\title{
NOETHER INEQUALITY FOR A NEF AND BIG DIVISOR ON A SURFACE
}

\author{
DONG-KWAN SHIN
}

\begin{abstract}
For a nef and big divisor $D$ on a smooth projective surface $S$, the inequality $h^{0}\left(S, \mathcal{O}_{S}(D)\right) \leq D^{2}+2$ is well known. For a nef and big canonical divisor $K_{S}$, there is a better inequality $h^{0}\left(S, \mathcal{O}_{S}\left(K_{S}\right)\right) \leq$ $\frac{1}{2} K_{S}{ }^{2}+2$ which is called the Noether inequality. We investigate an inequality $h^{0}\left(S, \mathcal{O}_{S}(D)\right) \leq \frac{1}{2} D^{2}+2$ like Clifford theorem in the case of a curve. We show that this inequality holds except some cases. We show the existence of a counter example for this inequality. We prove also the base-locus freeness of the linear system in the exceptional cases.
\end{abstract}

Throughout this paper, we are working over the complex number field $\mathbb{C}$.

For a nef and big divisor $D$ on a smooth projective surface $S$, the inequality $h^{0}\left(S, \mathcal{O}_{S}(D)\right) \leq D^{2}+2$ is well known. (See [2] p.173) When $S$ is a minimal projective surface of general type, the canonical divisor $K_{S}$ is nef and big. In this case, we have the better inequality which is called the Noether inequality:

$$
h^{0}\left(S, \mathcal{O}_{S}\left(K_{S}\right)\right) \leq \frac{1}{2} K_{S}^{2}+2 .
$$

The Noether inequality has played the important role to understand the geography of surfaces of general type. Now, if possible, we are going to try to generalize this inequality to an arbitrary nef and big divisor $D$ on a surface $S$. Thus, we investigate the inequality:

$$
h^{0}\left(S, \mathcal{O}_{S}(D)\right) \leq \frac{1}{2} D^{2}+2 .
$$

In the case of a curve, there is already Clifford theorem for a special divisor. We show that the inequality $(*)$ holds except some cases. We show the existence of such exceptional cases in which the inequality $(*)$ does not hold. We describe also the exceptional cases. For an example, when $h^{0}\left(S, \mathcal{O}_{S}(D)\right) \geq \frac{1}{2} D^{2}+2$, the linear system $|D|$ in the exceptional case has a very good property like the baselocus freeness. Using our results, we can prove again the Noether inequality for a canonical divisor on a surface of general type and the well known results about a nef divisor on a K3-surface.

Received February 28, 2007; Revised May 24, 2007.

2000 Mathematics Subject Classification. 14E05, 14 J99.

Key words and phrases. linear system, Noether inequality, nef and big divisor.

This paper was supported by KOSEF ABRL R14-2002-007-01001-0. 
We are going to use the following notations throughout.

Let $S$ be a smooth projective surface with a canonical divisor $K_{S}$. We denote the genus and the algebraic Euler characteristic of $S$ by $p_{g}(S)$ and $\chi\left(\mathcal{O}_{S}\right)$ respectively. Denote by $\Phi_{|D|}$ the rational map associated to the complete linear system $|D|$ when $D$ is a divisor on $S$. Denote the dimension of $H^{i}\left(S, \mathcal{O}_{S}(D)\right)$ by $h^{i}\left(S, \mathcal{O}_{S}(D)\right)$. If the base locus of $|D|$ is not empty, then there is a resolution of the base locus $\psi: S^{\prime} \rightarrow S$ by successive blowing-ups along smooth centers such that $\gamma=\Phi_{|D|} \circ \psi$ is a morphism. Let $\gamma=\beta \circ \alpha$ be the Stein factorization. Denote by $a$ the degree of the image $W$ of $\Phi_{|D|}$ in $\mathbf{P}^{h^{0}\left(S, \mathcal{O}_{S}(D)\right)-1}$. Denote by $b$ the degree of $\beta$.

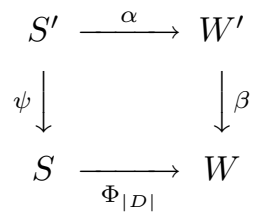

Thus, we have

$$
\begin{aligned}
D & =D_{M}+Z \\
\psi^{*} D & =D^{\prime}+Z^{\prime},
\end{aligned}
$$

where $Z$ (respectively, $Z^{\prime}$ ) is the fixed part of $|D|$ (respectively, $\left|\psi^{*} D\right|$ ), and $\left|D_{M}\right|$ (respectively, $\left|D^{\prime}\right|$ ) is the moving part of $|D|$ (respectively, $\left|\psi^{*} D\right|$ ). Moreover, the base locus of $\left|D^{\prime}\right|$ is empty. Also, we have $K_{S^{\prime}}=\psi^{*} K_{S}+E$, where $E$ is the effective divisor supported on the exceptional locus.

Since we are not interested in the case $h^{0}\left(S, \mathcal{O}_{S}(D)\right)=1$, we assume $h^{0}(S$, $\left.\mathcal{O}_{S}(D)\right) \geq 2$. Now, we are going to divide our problem into two cases - when $\operatorname{dim} \operatorname{Im} \Phi_{|D|}=2$ and when $\operatorname{dim} \operatorname{Im} \Phi_{|D|}=1$.

The following theorem is well known.

Theorem 1. Let $S$ be a nondegenerate surface in $\mathbf{P}^{n}$ with a degree $n-1$. Then $S$ is one of the following:
(a) a quadric surface in $\mathbf{P}^{3}$
(b) the Veronese surface in $\mathbf{P}^{5}$
(c) a rational normal scroll
(d) a cone over a rational normal twisted curve.

Proposition 1. Let $D$ be a nef and big divisor on a smooth projective surface S. If $\operatorname{dim} \operatorname{Im} \Phi_{|D|}=2$, then the following holds.

$$
h^{0}\left(S, \mathcal{O}_{S}(D)\right) \leq \max \left\{\frac{1}{2} \mathrm{D}^{2}+2, \frac{1}{2} \mathrm{D}^{2}-\frac{1}{2} \mathrm{~K}_{\mathrm{S}} \cdot \mathrm{D}_{\mathrm{M}}+1\right\} \text {. }
$$


Proof. We have

$$
\begin{aligned}
D^{2}=\psi^{*} D^{2} & =\psi^{*} D \cdot\left(D^{\prime}+Z^{\prime}\right) \\
& \geq \psi^{*} D \cdot D^{\prime} \\
& =\left(D^{\prime}+Z^{\prime}\right) \cdot D^{\prime} \\
& \geq{D^{\prime 2}}^{2} .
\end{aligned}
$$

Let $G$ be a general member of $\left|D^{\prime}\right|$. Since $\operatorname{dim} \operatorname{Im} \Phi_{\left|\mathrm{D}^{\prime}\right|}=2, G$ is a smooth irreducible curve on $S^{\prime}$.

Consider the following exact sequence:

$$
0 \rightarrow \mathcal{O}_{S^{\prime}} \rightarrow \mathcal{O}_{S^{\prime}}\left(D^{\prime}\right) \rightarrow \mathcal{O}_{G}\left(\left.D^{\prime}\right|_{G}\right) \rightarrow 0 .
$$

From this exact sequence, we have

$$
h^{0}\left(S^{\prime}, \mathcal{O}_{S^{\prime}}\left(D^{\prime}\right)\right)-1 \leq h^{0}\left(G, \mathcal{O}_{G}\left(\left.D^{\prime}\right|_{G}\right)\right) .
$$

Let's consider the following two cases.

First, if $h^{1}\left(G, \mathcal{O}_{G}\left(\left.D^{\prime}\right|_{G}\right)\right) \neq 0$, then we have $h^{0}\left(G, \mathcal{O}_{G}\left(\left.D^{\prime}\right|_{G}\right)\right)-1 \leq \frac{1}{2}{D^{\prime}}^{2}$ by Clifford theorem. Hence, from (2), we have

$$
h^{0}\left(S^{\prime}, \mathcal{O}_{S^{\prime}}\left(D^{\prime}\right)\right)-2 \leq h^{0}\left(G, \mathcal{O}_{G}\left(\left.D^{\prime}\right|_{G}\right)\right)-1 \leq \frac{1}{2} D^{\prime 2} .
$$

Since $h^{0}\left(S, \mathcal{O}_{S}(D)\right)=h^{0}\left(S^{\prime}, \mathcal{O}_{S^{\prime}}\left(D^{\prime}\right)\right)$, we have from (1) and (3),

$$
h^{0}\left(S, \mathcal{O}_{S}(D)\right) \leq \frac{1}{2} D^{\prime 2}+2 \leq \frac{1}{2} D^{2}+2 .
$$

Second, if $h^{1}\left(G, \mathcal{O}_{G}\left(\left.D^{\prime}\right|_{G}\right)\right)=0$, then by Riemann-Roch theorem, we have

$$
\begin{aligned}
h^{0}\left(G, \mathcal{O}_{G}\left(\left.D^{\prime}\right|_{G}\right)\right) & =1+{D^{\prime 2}}^{2}-p_{g}(G) \\
& =1+{D^{\prime 2}}^{2}-\left(1+\frac{D^{\prime 2}+K_{S^{\prime}} \cdot D^{\prime}}{2}\right) \\
& =\frac{1}{2} D^{\prime 2}-\frac{1}{2} K_{S^{\prime}} \cdot D^{\prime} .
\end{aligned}
$$

We have $K_{S^{\prime}} \cdot D^{\prime}=\left(\psi^{*} K_{S}+E\right) \cdot D^{\prime}=K_{S} \cdot D_{M}+E \cdot D^{\prime}$ since $\psi^{*} K_{S} \cdot D^{\prime}=K_{S} \cdot D_{M}$. Hence

$$
-K_{S^{\prime}} \cdot D^{\prime}=-K_{S} \cdot D_{M}-E \cdot D^{\prime} \leq-K_{S} \cdot D_{M}
$$

since $E \cdot D^{\prime} \geq 0$. From (1) and (5), we have

$$
h^{0}\left(G, \mathcal{O}_{G}\left(\left.D^{\prime}\right|_{G}\right)\right)=\frac{1}{2} D^{\prime 2}-\frac{1}{2} K_{S^{\prime}} \cdot D^{\prime} \leq \frac{1}{2} D^{2}-\frac{1}{2} K_{S} \cdot D_{M} .
$$

Hence, from (2), we have

$$
h^{0}\left(S, \mathcal{O}_{S}(D)\right)-1 \leq h^{0}\left(G, \mathcal{O}_{G}\left(\left.D^{\prime}\right|_{G}\right)\right) \leq \frac{1}{2} D^{2}-\frac{1}{2} K_{S} \cdot D_{M} .
$$


Combining (4) and (6), we have that

$$
h^{0}\left(S, \mathcal{O}_{S}(D)\right) \leq \max \left\{\frac{1}{2} \mathrm{D}^{2}+2, \frac{1}{2} \mathrm{D}^{2}-\frac{1}{2} \mathrm{~K}_{\mathrm{S}} \cdot \mathrm{D}_{\mathrm{M}}+1\right\} .
$$

Theorem 2. Let $D$ be a nef and big divisor on a smooth projective surface $S$ with $\kappa(S) \geq 0$, where $\kappa(S)$ is the Kodaira dimension of $S$. Assume that $\operatorname{dim} \operatorname{Im} \Phi_{|\mathrm{D}|}=2$. Then $h^{0}\left(S, \mathcal{O}_{S}(D)\right) \leq \frac{1}{2} D^{2}+2$.

Proof. Since $\kappa(S) \geq 0$, there is a multiple of $K_{S}$ which is effective. Hence $K_{S} \cdot D_{M}$ in Proposition 1 is nonnegative. Thus,

$$
\frac{1}{2} D^{2}-\frac{1}{2} K_{S} \cdot D_{M}+1 \leq \frac{1}{2} D^{2}+1
$$

Therefore we have

$$
h^{0}\left(S, \mathcal{O}_{S}(D)\right) \leq \frac{1}{2} D^{2}+2 .
$$

Corollary 1. If the equality holds in Theorem 2, i.e., $h^{0}\left(S, \mathcal{O}_{S}(D)\right)=\frac{1}{2} D^{2}+2$, then we have the following:

(a) $|D|$ is base-locus and fixed-locus free.

(b) $S$ is birational onto a K3 surface or $\Phi_{|D|}$ is generically 2:1 onto a surface which is described in Theorem 1.

Proof. From the proof of Proposition 1, we have

$$
\frac{1}{2} D^{2}+2=h^{0}\left(S, \mathcal{O}_{S}(D)\right) \leq \frac{1}{2} D^{\prime 2}+2 \leq \frac{1}{2} D^{2}+2 .
$$

Thus we have $D^{\prime 2}=D^{2}$. Then from (1), we have $\psi^{*} D \cdot Z^{\prime}=0=D^{\prime} \cdot Z^{\prime}$. Since $\psi^{*} D \cdot Z^{\prime}=D^{\prime} \cdot Z^{\prime}+Z^{\prime 2}$, we have $Z^{\prime 2}=0$. Hence, by Hodge Index Theorem, $Z^{\prime}=0$. It means that $|D|$ is base-locus and fixed-locus free because $Z^{\prime}$ comes from the fixed part $Z$ and the base locus of $|D|$. Also we have $D^{2}=a b$. Since $2 h^{0}\left(S, \mathcal{O}_{S}(D)\right)-4=D^{2}$, we have

$$
\left(h^{0}\left(S, \mathcal{O}_{S}(D)\right)-2\right) b \leq a b=D^{2}=2 h^{0}\left(S, \mathcal{O}_{S}(D)\right)-4 .
$$

Hence $b \leq 2$.

For (b), we have $h^{1}\left(G, \mathcal{O}_{G}\left(\left.D\right|_{G}\right)\right) \neq 0$. If not, from the proof of Proposition 1 , we have $h^{0}\left(S, \mathcal{O}_{S}(D)\right) \leq \frac{1}{2} D^{2}-\frac{1}{2} K_{S} \cdot D_{M}+1$. But it is impossible because $h^{0}\left(S, \mathcal{O}_{S}(D)\right)=\frac{1}{2} D^{2}+2$ and $K_{S} \cdot D_{M} \geq 0$. Thus, we have $h^{0}\left(G, \mathcal{O}_{G}\left(\left.D\right|_{G}\right)\right)-1=$ $\frac{1}{2} D^{2}$ from $(3)$ since $h^{0}\left(S, \mathcal{O}_{S}(D)\right)=\frac{1}{2} D^{2}+2$. It means that the equality holds in Clifford theorem since $h^{1}\left(G, \mathcal{O}_{G}\left(\left.D\right|_{G}\right)\right) \neq 0$. Hence we have the following two cases according to Clifford theorem.

(i) $\left.D\right|_{G}=K_{G}$ or

(ii) $G$ is hyperelliptic and $\left.D\right|_{G}$ is a positive multiple of a hyperelliptic divisor. 
In the case of (i), we have $\left.K_{S}\right|_{G}=0$ from the adjunction formula. Let $f: S \rightarrow S_{\min }$ be a minimal model of $S$. Then $f^{*} K_{S_{\min }} \cdot D=0$. By Hodge index theorem, $K_{S_{\min }}=0$ since some multiple of $K_{S_{\min }}$ is effective. For a simplicity, we may assume that $S$ is minimal. From the exact sequence

$$
0 \rightarrow \mathcal{O}_{S} \rightarrow \mathcal{O}_{S}(D) \rightarrow \mathcal{O}_{G}\left(\left.D\right|_{G}\right) \rightarrow 0
$$

we have the following exact sequence

$$
0 \rightarrow h^{0}\left(S, \mathcal{O}_{S}\right) \rightarrow h^{0}\left(S, \mathcal{O}_{S}(D)\right) \rightarrow h^{0}\left(G, \mathcal{O}_{G}\left(\left.D\right|_{G}\right)\right) \rightarrow h^{1}\left(S, \mathcal{O}_{S}\right) \rightarrow 0,
$$

since $h^{1}\left(S, \mathcal{O}_{S}(D)\right)=0$ by Kawamata-Viehweg vanishing theorem. By the dimension computation, $h^{0}\left(S, \mathcal{O}_{S}(D)\right)=h^{0}\left(G, \mathcal{O}_{G}\left(\left.D\right|_{G}\right)\right)+1$. Hence we have $h^{1}\left(S, \mathcal{O}_{S}\right)=0$ from the exact sequence. It means that $S$ is a K3 surface.

In the case of (ii), the degree $b$ of $\Phi_{|D|}$ must be 2 . If $b=1$ then we have $\left.D\right|_{G}=K_{G}$ which is already covered in the case (i). Since $b=2$, the degree $a$ of the image of $\Phi_{|D|}$ is $h^{0}\left(S, \mathcal{O}_{S}(D)\right)-2$. Then $\Phi_{|D|}$ is generically $2: 1$ onto a surface which is described in Theorem 1.

Remark 1. In Theorem 2, the condition $\kappa(S) \geq 0$ is necessary. In the projective plane $\mathbb{P}^{2}$, if $D$ is a hyperplane section $H, K_{\mathbb{P}^{2}} \cdot H=-3$ and

$$
h^{0}\left(\mathbb{P}^{2}, \mathcal{O}_{\mathbb{P}^{2}}(H)\right)=3>\frac{1}{2}+2=\frac{H^{2}}{2}+2 .
$$

Now, let's investigate the remaining case, i.e., when $\operatorname{dim} \operatorname{Im} \Phi_{|D|}=1$. In this case, unfortunately we have a counter example even on a surface of general type. Thus, we are going to describe the possible exceptional case in which the inequality $(*)$ does not hold.

Theorem 3. There exist a surface $S$ of general type and a nef and big divisor on $S$ which does not satisfy the inequality $(*)$.

Proof. We know that there is a smooth projective threefold $X$ of general type such that its canonical divisor $K_{X}$ is ample, $\operatorname{dim} \operatorname{Im} \Phi_{\left|K_{X}\right|}=2, p_{g}(X)=$ $\frac{3}{4} K_{X}{ }^{3}+\frac{10}{4}$ and $p_{g}(X) \geq 7$ (see M. Kobayashi [3, Proposition (3.2)]). Resolve the base-locus of $\left|K_{X}\right|$, i.e., $\psi: X^{\prime} \rightarrow X$ such that $\Phi_{\left|K_{X}\right|} \circ \psi$ is a morphism. Then we have

$$
\left|\psi^{*} K_{X}\right|=\left|D_{M}\right|+Z_{X},
$$

where $\left|D_{M}\right|$ is the moving part of $\left|\psi^{*} K_{X}\right|$ and base-locus free. Clearly a general member $S$ of $\left|D_{M}\right|$ is a smooth projective surface of general type since $p_{g}(X) \geq$ 7. From the exact sequence

$$
0 \rightarrow \mathcal{O}_{X^{\prime}}\left(Z_{X}\right) \rightarrow \mathcal{O}_{X^{\prime}}\left(\psi^{*} K_{X}\right) \rightarrow \mathcal{O}_{S}\left(\left.\psi^{*} K_{X}\right|_{S}\right) \rightarrow 0,
$$

we have $p_{g}(X) \leq h^{0}\left(S, \mathcal{O}_{S}\left(\left.\psi^{*} K_{X}\right|_{S}\right)\right)+1 .\left.\psi^{*} K_{X}\right|_{S}$ is nef and big on $S$. Suppose that the divisor $\left.\psi^{*} K_{X}\right|_{S}$ on $S$ satisfy the inequality $(*) \cdot h^{0}\left(S, \mathcal{O}_{S}\left(\left.\psi^{*} K_{X}\right|_{S}\right)\right) \leq$ 
$\left.\frac{1}{2} \psi^{*} K_{X}\right|_{S} ^{2}+2$ by our assumption. Hence

$$
p_{g}(X) \leq\left.\frac{1}{2} \psi^{*} K_{X}\right|_{S} ^{2}+3 \leq \frac{1}{2} K_{X}^{3}+3
$$

Since $p_{g}(X) \geq 7, K_{X}{ }^{3} \geq 6$. However, $\frac{1}{2} K_{X}{ }^{3}+3<\frac{3}{4} K_{X}{ }^{3}+\frac{10}{4}$ since $K_{X}{ }^{3} \geq 6$. It is a contradiction.

To describe the possible exceptional cases, we are going to use the following notations.

Assume that $\operatorname{dim} \operatorname{Im} \Phi_{|D|}=1$. Let $C^{\prime}$ be a general fiber of $\alpha$ in the Stein factorization.

$$
D^{2}=\psi^{*} D^{2}=\left(a b C^{\prime}+Z^{\prime}\right) \cdot \psi^{*} D \geq a b \psi^{*} D \cdot C^{\prime}=a b c,
$$

where $c=\psi^{*} D \cdot C^{\prime}$. Thus, we have $a \leq \frac{D^{2}}{b c}$. Since $W$ is nondegenerate, we have $h^{0}\left(S, \mathcal{O}_{S}(D)\right)-1 \leq a$. Hence we have

$$
h^{0}\left(S, \mathcal{O}_{S}(D)\right)-1 \leq a \leq \frac{D^{2}}{b c}
$$

Lemma 1. Suppose that $\operatorname{dim} \operatorname{Im} \Phi_{|D|}=1$ and the inequality $(*)$ does not hold. Then $b c=1$.

Proof. If $b c \geq 2$, then we have $h^{0}\left(S, \mathcal{O}_{S}(D)\right)-1 \leq \frac{1}{2} D^{2}$ from (8), so the inequality $(*)$ holds. Hence $b c=1$.

The fact $b c=1$ means that $\alpha: S^{\prime} \rightarrow W^{\prime}$ is a morphism of $S^{\prime}$ onto a smooth curve $W^{\prime}$ with connected fiber and $W^{\prime}$ is birational to $W$. Hence we may assume that $W$ is smooth.

Proposition 2. Let $D$ be a nef and big divisor on a smooth projective surface S. Suppose that $\operatorname{dim} \operatorname{Im} \Phi_{|D|}=1$ and the inequality $(*)$ does not hold. Then we have

(a) $|D|$ is base-locus free and has non-zero fixed component $Z$.

(b) D cannot be numerically equivalent to a sum of two nef and big divisors.

(c) $p_{g}(W)=a-h^{0}\left(S, \mathcal{O}_{S}(D)\right)+1$.

Proof. For a proof of (a),

$$
\begin{aligned}
1=c & =\psi^{*} D \cdot C^{\prime} \\
& =D \cdot C \\
& =(a C+Z) \cdot C \\
& =a C \cdot C+Z \cdot C,
\end{aligned}
$$

where $C=\psi_{*} C^{\prime}$. Since $h^{0}\left(S, \mathcal{O}_{S}(D)\right) \geq 3, a \geq h^{0}\left(S, \mathcal{O}_{S}(D)\right)-1 \geq 2$. Thus we have $C^{2}=0$ and $C \cdot Z=1$ since $C^{2} \geq 0$. It means $D_{M}{ }^{2}=a C \cdot a C=0$. Hence the linear system $|D|$ is base point-free and must have a non-zero fixed component $Z$ with $C \cdot Z=1$. 
For a proof of (b), assume $D=D_{1}+D_{2}$, where $D_{1}, D_{2}$ are nef and big. $c=\psi^{*} D \cdot C^{\prime}=\psi^{*} D_{1} \cdot C^{\prime}+\psi^{*} D_{2} \cdot C^{\prime}$. Since each $\psi^{*} D_{i} \cdot C^{\prime} \geq 1$ for $i=1,2$, we have $c \geq 2$, but $\psi^{*} D \cdot C^{\prime}=c=1$ which is explained in Lemma 1 .

For a proof of (c), let's denote $\Phi_{|D|}$ by $\Phi$ for a simplicity. Then we have

$$
h^{0}\left(W, \Phi_{*} \mathcal{O}_{S}\left(D_{M}\right)\right)-h^{1}\left(W, \Phi_{*} \mathcal{O}_{S}\left(D_{M}\right)\right)=1+a-p_{g}(W) .
$$

If $h^{1}\left(W, \Phi_{*} \mathcal{O}_{S}\left(D_{M}\right)\right) \neq 0$, then we have, by Clifford theorem,

$$
h^{0}\left(S, \mathcal{O}_{S}(D)\right)=h^{0}\left(W, \Phi_{*} \mathcal{O}_{S}\left(D_{M}\right)\right) \leq \frac{1}{2} a+1 \leq \frac{1}{2} D^{2}+1,
$$

so the inequality $(*)$ holds. Hence $h^{1}\left(W, \Phi_{*} \mathcal{O}_{S}\left(D_{M}\right)\right)=0$, which means

$$
h^{0}\left(S, \mathcal{O}_{S}(D)\right)=1+a-p_{g}(W) .
$$

Remark 2. Recall that $D \cdot C=1$ and $C^{2}=0$ in the proof of Proposition 2 if the inequality $(*)$ does not hold. The original Noether inequality for a surface of general type comes from Theorem 2 and Proposition 2. If Noether inequality fails to hold, then $\operatorname{dim} \operatorname{Im} \Phi_{\left|K_{S}\right|}=1$ and $K_{S} \cdot C=1, C^{2}=0$. By a genus formula, we have $K_{S} \cdot C \equiv C^{2}(\bmod 2)$. It contradicts. Thus, the original Noether inequality holds true.

Remark 3 . Let $S$ be a minimal surface with $\kappa(S) \geq 0$. The inequality $(*)$ holds clearly in the following cases:

(1) The inequality $(*)$ holds when $D$ is a canonical divisor.

(2) The inequality $(*)$ holds for the adjoint linear system $\left|K_{S}+D\right|$.

(3) On a K3 surface, by a vanishing theorem, $h^{0}\left(S, \mathcal{O}_{S}(D)\right)=\frac{1}{2} D^{2}+2$.

(4) For a positive multiple $n D(n \geq 2)$, the inequality $(*)$ holds by Theorem 2 and Proposition 2.

Remark 4. If $S$ is a K3 surface and $D$ is a nef divisor on $S$, then we may have the well known results about a nef divisor on a K3 surface from the following theorem. (For detail matters, see B. Saint-Donat [4]).

Lemma 2. Let $D$ be a nef divisor with $D^{2}=0$ on a smooth projective surface. Suppose $h^{0}\left(S, \mathcal{O}_{S}(D)\right) \geq 2$. Then $D$ is numerically equivalent to a positive multiple of a curve $C$ with $C^{2}=0$.

Proof. Since $D^{2}=0$, the image of $\Phi_{|D|}$ must be a curve. Thus, we have

$$
D^{2}=D \cdot(a b C+Z)=a b D \cdot C+D \cdot Z,
$$

which is explained just above Lemma 1 and in the proof of Proposition 2. Since $D$ is nef, we have $D \cdot C=0$ and $D \cdot Z=0$. Since $C^{2} \geq 0$, from these, we have $C^{2}=C \cdot Z=Z^{2}=0$ as explained in the proof of Proposition 2. Hence $D$ is base-locus free and $Z=0$. If not, then we must have a fiber of the form $C_{0}+Z$ with $C_{0} \cdot Z>0$. But it contradicts the fact that $C \cdot Z=0$.

Hence $D$ is numerically equivalent to a positive multiple of $C$ with $C^{2}=$ 0 . 
Theorem 4. Let $D$ be a nef divisor on a surface $S$ with $\kappa(S) \geq 0$. If $h^{0}\left(S, \mathcal{O}_{S}(D)\right) \geq \frac{1}{2} D^{2}+2$, then we have the following.

(a) $|D|$ is base-locus free.

(b) Suppose $D^{2}=0 . D$ is numerically equivalent to a positive multiple of a curve $C$ with $C^{2}=0$.

(c) Suppose $D^{2}>0$. If $\operatorname{dim} \operatorname{Im} \Phi_{|D|}=2$, then the degree of $\Phi_{|D|}$ is at most 2.

(d) Suppose $D^{2}>0 .|D|$ has a nonzero fixed part if and only if the image of $\Phi_{|D|}$ is a curve.

Proof. Proof of this theorem comes clearly from Corollary 1, Proposition 2 and Lemma 2.

\section{References}

[1] W. Barth, C. Peters and A. Van de Ven, Compact Complex Surfaces, Springer-Verlag, Berlin-Heidelberg-New-York, 1984.

[2] P. Griffiths and J. Harris, Principles of Algebraic Geometry, Wiley, New-York, 1978.

[3] M. Kobayashi, On Noether's inequality for threefolds, J. Math. Soc. Japan 44 (1992), no. $1,145-156$.

[4] B. Saint-Donat, Projective models of K3 surfaces, Amer. J. of Math. 96 (1974), no. 4, 602-639.

Department of Mathematics

KonkuK UNIVERSITY

SEOul 143-701, Korea

E-mail address: dkshin@konkuk.ac.kr 